\title{
A FORMULA FOR STIEFEL-WHITNEY HOMOLOGY CLASSES
}

\author{
RICHARD Z. GOLDSTEIN ${ }^{1}$ AND EDWARD C. TURNER
}

\begin{abstract}
The purpose of this paper is to define for mod 2 Euler spaces a formula which enables one to compute the Stiefel-Whitney homology classes in the original triangulation without passing to the first barycentric subdivision.
\end{abstract}

The purpose of this paper is to define for mod 2 Euler spaces a formula which enables one to compute the Stiefel-Whitney homology classes in the original triangulation without passing to the first barycentric subdivision. The formula has a somewhat tenuous connection to the Steenrod reduced squares. In the case when we are dealing with a smooth triangulation, the $\mathrm{Wu}$ formulae [7] and the Whitney theorem [4] establish such a connection. The authors would like to thank S. Halperin and D. Toledo for a copy of their preprint [5]; the use of their map $\phi$ (see \$2) simplifies an earlier proof of the main theorem. The homology theory used is that based on infinite chains.

1. Statement of the theorem. Let $K$ be a finite-dimensional, locally finite simplicial complex. $K$ is said to be a mod 2 Euler space if the link of every simplex in $K$ has even Euler characteristic [9]. The $p$ th Stiefel-Whitney class of $K$, denoted $\omega_{p}(K)$, is the $p$-dimensional mod 2 homology class which has a representative, the $p$-dimensional chain consisting of all $p$-simplexes in the first barycentric subdivision of $K$-this chain is a cycle for each $p$ iff $K$ is a mod 2 Euler space.

From now on we assume that $K$ is given an ordering of its vertices and any representation of a simplex in $K$ is written with its vertices in increasing order. We now recall a definition due to Steenrod [8]. Let $s$ be a $p$-simplex in $K$, say $s=\left\langle v_{0}, v_{1}, \ldots, v_{p}\right\rangle$. Let $t$ be another simplex which has $s$ as a face; i.e., $s \subset t(s$ may be equal to $t)$. Let

$B_{-1}=$ set of vertices of $t$ less than $v_{0}$,

$B_{0}=$ set of vertices of $t$ strictly between $v_{0}$ and $v_{1}$,

$B_{m}=$ set of vertices of $t$ strictly between $v_{m}$ and $v_{m+1}$,

$B_{p}=$ set of vertices of $t$ greater than $v_{p}$.

We say that $s$ is regular in $t$, if $\#\left(B_{m}\right)=0$ for every odd $m$. Let $\partial_{p}(t)$ denote the mod 2 chain which consists of all $p$-dimensional simplexes $s$ in $t$ so that $s$

Presented to the Society, April 11, 1975; received by the editors April 28, 1975 and, in revised form, November 10, 1975.

AMS (MOS) subject classifications (1970). Primary 57C99; Secondary 57D20.

Key words and phrases. Stiefel-Whitney homology class, mod 2 Euler space.

${ }^{1}$ Partially supported by the Humboldt Stiftung. 
is regular in $t$. The following is the main result in this paper.

THEOREM. $\sum_{\operatorname{dim} t \geqslant p} \partial_{p}(t)$ is a chain which represents $\omega_{p}(K)$.

2. Proof of the main theorem. We define a simplicial map $\phi: K^{\prime} \rightarrow K$ in the following way. Pick a simplex $s$ in $K$ and let $l(s)$ be the smallest vertex in $s$; map the barycenter of $s$ to $l(s)$. This defines a simplicial map $\phi: K^{\prime} \rightarrow K$ which induces the identity in homology. To establish the theorem it suffices to show that the number of $p$-simplexes in $K^{\prime}$ which map onto a given $p$-simplex $s$ in $K$ is congruent mod 2 to the number of simplexes $t$, so that $s$ is regular in $t$. However a $p$-simplex $s^{\prime}$ in $K^{\prime}$ maps onto $s$ only if $s$ is a face of the carrier in $K$ of $s^{\prime}$; hence the theorem is a consequence of the following

Lemma. Let $\phi: K^{\prime} \rightarrow K$ be defined as above and let $s$ be a face of $t$; then the number of p-simplexes whose carrier is $t$ and which map onto $s$ is

(i) odd if $s$ is regular in $t$,

(ii) even if $s$ is not regular in $t$.

Proof. If $B_{-1}$ is not empty then $s$ is not regular in $t$ and no simplex whose carrier is $t$ maps onto $s$. Hence we assume that $B_{-1}$ is empty. Now a $p$-simplex whose carrier is $t$ and whose image under $\phi$ is $s$ must look like the following:

$$
\left(\left\{v_{0}, B_{0}, v_{1}, B_{1}, \ldots, v_{p}, B_{p}\right\},\left\{v_{1}, B_{1}^{\prime}, v_{2}, \ldots, v_{p}, B_{p}^{\prime}\right\}, \ldots,\left\{v_{p}, B_{p}^{(p)}\right\}\right)
$$

where

$$
\begin{aligned}
B_{1}^{\prime} & \subset B_{1}, \\
B_{2}^{\prime \prime} & \subset B_{2}^{\prime} \subset B_{2}, \\
& \vdots \\
B_{p}^{(p)} & \subset \cdots \subset B_{p}^{\prime} \subset B_{p} .
\end{aligned}
$$

Denote by $c\left(B_{j}\right)$, the number of ways of choosing $j$ nondecreasing subsets of $B_{j}$; hence the number of $p$-simplexes whose image is $s$ is $c\left(B_{1}\right) \cdot c\left(B_{2}\right) \cdots$ - $c\left(B_{p}\right)$.

Proposition. (1) $c\left(B_{j}\right)=1$ if $B_{j}=\varnothing$,

(2) $c\left(B_{j}\right) \equiv 0(\bmod 2)$ if $B_{j} \neq \varnothing$ and $j$ is odd,

(3) $c\left(B_{j}\right) \equiv 1(\bmod 2)$ if $B_{j} \neq \varnothing$ and $j$ is even.

Proof. Part 1 follows from the fact that $B_{j}^{(k)}$ is always the null set.

Now when $m \geqslant 0$ we have that

$$
\begin{aligned}
\sum_{a=0}^{m} \sum_{b=0}^{a}\left(\begin{array}{l}
m \\
a
\end{array}\right)\left(\begin{array}{l}
a \\
b
\end{array}\right) & =\sum_{a=0}^{m}\left(\begin{array}{l}
m \\
a
\end{array}\right) \sum_{b=0}^{a}\left(\begin{array}{l}
a \\
b
\end{array}\right) \\
& \equiv\left(\begin{array}{c}
m \\
0
\end{array}\right)(\bmod 2) \equiv 1 \quad(\bmod 2) .
\end{aligned}
$$

Now in general

$$
c\left(B_{j}\right)=\sum_{n_{1}=0}^{n} \sum_{n_{2}=0}^{n_{1}} \cdots \sum_{n_{j}=0}^{n_{j-1}}\left(\begin{array}{c}
n \\
n_{1}
\end{array}\right)\left(\begin{array}{c}
n_{1} \\
n_{2}
\end{array}\right) \cdots\left(\begin{array}{c}
n_{j-1} \\
n_{j}
\end{array}\right) \text { where \# }\left(B_{j}\right)=n_{j} .
$$

Therefore, $\bmod 2$ we have that 
$c\left(B_{j}\right) \equiv 1$ when $j$ is even.

$c\left(B_{j}\right)=\sum_{n_{1}=0}^{n}\left(\begin{array}{c}n \\ n_{1}\end{array}\right) \equiv 0$ when $j$ is odd since $n>0$.

Now back to the proof of the lemma. When $s$ is regular in $t, B_{j}=\varnothing$ for $j$ odd; hence

$$
c\left(B_{1}\right) \cdot c\left(B_{2}\right) \cdots \cdots c\left(B_{p}\right) \equiv 1 \cdot 1 \cdots \cdot 1 \equiv 1 \bmod 2 .
$$

When $s$ is not regular in $t$, some $B_{j} \neq \varnothing$ for $j$ odd; hence $c\left(B_{j}\right) \equiv 0$ and the lemma is proven.

3. Some remarks on the formula. The above proof works verbatim in the case that the vertices are partially ordered in such a way that those of any simplex are linearly ordered, as in the barycentric subdivision ordered by dimension. It is not hard to see that in $K^{\prime}$, each $p$-simplex is a regular face of an odd number of simplexes, so that

$$
\sum_{\operatorname{dim}(t) \geqslant p} \partial_{p}(t)=\sum p \text {-simplexes of } K^{\prime}
$$

It follows that the $p$-skeleta of repeated barycentric subdivisions are homologous. (E. Akin has shown that $\omega_{p}(K)$ is a PL invariant [1].)

When $p=0$ it is tempting to call the coefficient of a vertex $v$ in

$$
\sum_{\operatorname{dim} t \geqslant 0} \partial_{0}(t)
$$

the local Euler number. The reason is two-fold: first the number of vertices whose coefficient is 1 is congruent $\bmod 2$ to $\chi(K)$. Secondly when $K$ is an immersed surface in $R^{3}$ and the ordering on the vertices is induced by projection on the $z$-axis, then the vertices with coefficient 1 are precisely the critical points of the function defined on $K$ by this projection. Thus we get that $\chi(K) \equiv$ number critical pts $(\bmod 2)$.

When $K$ is $n$-dimensional, then the ordering on the vertices induces an orientation on each simplex in $K$. Now when $s$ is an $(n-1)$-simplex and a proper face of $t$, we have that $s$ is regular in $t$ if and only if the orientation on $t$ induces the opposite orientation on $s$. Thus $\omega_{n-1}(K)$ has as a representative the sum of those $(n-1)$-simplexes whose orientation disagrees with an even number of $n$-simplexes of which it is a face (a simplex is always regular in itself). This last fact is exploited in [3].

In fact our formula can be derived from the work of Banchoff [2] and McCrory [6]. Given an ordering of the vertices of $K$ define a vertex map into $R^{m}$ by sending the vertex $j$ into $\left(j, j^{2}, \ldots, j^{m}\right)$. This defines a full map and some combinatorics applied to the description of the S.W. classes as given in [6] gives our formula.

\section{REFERENCES}

1. E. Akin, Stiefel-Whitney homology classes and bordism, Trans. Amer. Math. Soc. 205 (1975), 341-359. 
2. T. Banchoff, Stiefel-Whitney homology classes and singularities of projections for polyhedral manifolds, Proc. Sympos. Pure Math., vol. 27, Amer. Math. Soc., Providence, R.I., 1975.

3. R. Goldstein, $A$ Wu-type formula for $E$ (2) spaces, Compositio Math. 32 (1976), 33-39.

4. S. Halperin and D. Toledo, Stiefel-Whitney homology classes, Ann. of Math. (2) 96 (1972), 511-525. MR 47 \# 1072.

5. , The product formula for Stiefel-Whitney homology classes, Proc. Amer. Math. Soc. 48 (1975), 239-244.

6. C. McCrory, Euler singularities and homology and operations, Proc. Sympos. Pure Math., vol. 27, Amer. Math. Soc., Providence, R.I., 1975.

7. J. W. Milnor, Characteristic classes, Princeton Univ. Press, Princeton, N.J., 1974.

8. N. Steenrod, Products of cocycles and extensions of mappings, Ann. of Math. (2) 48 (1947), 290-320. MR 9, 154.

9. D. Sullivan, Combinatorial invariants of analytic spaces, Proc. Liverpool Singularities Sympos., I (1969/70), Lecture Notes in Math., vol. 192, Springer-Verlag, Berlin, 1971. MR 43 \# 4063 .

Mathematisches Institut, Universität Würzburg, Würzburg, Federal Republic of GERMANY

Department of Mathematics, State University of New York, Albany, New York 1222 